\title{
POINCARÉ-MELNIKOV-ARNOLD METHOD FOR TWIST MAPS
}

\author{
AMADEU DELSHAMS AND RAFAEL RAMíREZ-ROS
}

\section{Introduction}

A general theory for perturbations of an integrable planar map with a separatrix to a hyperbolic fixed point has been developed in a previous lecture [5]. The splitting of the perturbed invariant curves was measured, in first order with respect to the parameter of perturbation, by means of a periodic Melnikov function $M$ defined on the unperturbed separatrix. In the case of planar twist maps, $M$ has zero mean and therefore there exists a periodic function $L$ (called the Melnikov potential) such that $M=L^{\prime}$. Consequently, if $L$ is not identically constant (respectively, has non-degenerate critical points), the separatrix splits (respectively, the perturbed curves cross transversely).

The aim of this lecture is to present a similar theory for more dimensions. The natural frame is to consider twist maps on cotangent bundles. Once the suitable definition of unperturbed separatrix has been introduced (a non-trivial problem in the high-dimensional case), a scalar function $L$ can be defined on it, in such a way that $L$ verifies the same properties than in the planar case. The derivation of $L$ is easily related to variational principles, and the property of being a scalar function instead of a vectorial function like the classical Melnikov function, makes it more useful for computations and geometrical understanding. Even more, it allows the application of Morse theory to establish the minimal number of transverse homoclinic orbits.

The results to be presented in this lecture are valid for exact symplectic maps on arbitrary exact symplectic manifolds, that is, the twist character is not essential. We have restricted ourselves to twist maps only for simplicity. Full details of the ideas presented here are contained in [4], where another more general situation (i.e., the exact symplectic case) is studied. Related ideas can be found in $[1,15,14,8,7]$.

\section{The maps}

A twist map $F$ is a map from a connected subset $U$ of the cotangent bundle of a manifold $\mathcal{M}$ (which can be non-compact) into $U$, which comes equipped with a twist generating function $\mathcal{L}: \mathcal{M} \times \mathcal{M} \rightarrow \mathbb{R}$ that satisfies

$$
F^{*}(y \mathrm{~d} x)-y \mathrm{~d} x=Y \mathrm{~d} X-y \mathrm{~d} x=\mathrm{d} \mathcal{L}(x, X), \quad(X, Y)=F(x, y),
$$

where $(x, y)$ are any cotangent coordinates on $T^{*} \mathcal{M}$, that is, $x$ are coordinates on $\mathcal{M}$, extended to coordinates $(x, y)$ in the obvious way. The symplectic form $\omega_{0}$ on $T^{*} \mathcal{M}$ reads 
as $\omega_{0}=\mathrm{d} x \wedge \mathrm{d} y$ in cotangent coordinates. This can also be written in a coordinate free manner. Given $\mathcal{L}$, one can retrieve the map (at least implicitly) from $y=-\partial_{1} \mathcal{L}(x, X)$, and $Y=\partial_{2} \mathcal{L}(x, X)$. This can be done globally (i.e., $\left.U=T^{*} \mathcal{M}\right)$ only when $\mathcal{M}$ is diffeomorphic to a fiber of $T^{*} \mathcal{M}$, for example when $\mathcal{M}$ is the covering space of $\mathbb{T}^{n}$ or a manifold of constant negative curvature.

Finally, let us denote by $\pi: T^{*} \mathcal{M} \rightarrow \mathcal{M}$ the canonical projection.

\section{The theory}

Assume now that we are given a smooth twist diffeomorphism $F_{0}$ on the cotangent bundle $T^{*} \mathcal{M}$. Let $\mathcal{L}_{0}$ be its twist generating function. We assume that there exists a hyperbolic fixed point $z_{0}^{\infty}$ of $F_{0}$, such that its $n$-dimensional (unstable and stable) invariant manifolds $\mathcal{W}_{0}^{\mathrm{u}, \mathrm{s}}$ are doubled, that is, they coincide: $\mathcal{W}:=\mathcal{W}_{0}^{\mathrm{u}}=\mathcal{W}_{0}^{\mathrm{s}}$

In the planar case, the separatrix consists of the intersection of the invariant curves, except for the hyperbolic fixed point, which is the only point where such invariant manifolds are not submanifolds of the cotangent bundle. In the high-dimensional case, the situation is more complicated. We can consider three topologies on the set $\mathcal{W}$ : the one induced by the inclusion $\mathcal{W} \subset T^{*} \mathcal{M}$, and the two ones induced by the inclusions $\mathcal{W} \subset \mathcal{W}_{0}^{\mathrm{u}, \mathrm{s}}$. We first define the bifurcation set $\Sigma$ of this problem as the subset of $\mathcal{W}$ formed by the points such that the three topologies do not coincide. Then, the separatrix $\Lambda$ is defined as its complementary in $W$, i.e.,

$$
\Lambda:=\mathcal{W} \backslash \Sigma \text {. }
$$

With this definition, it turns out that $\Lambda$ is a doubly asymptotic exact submanifold of $T^{*} \mathcal{M}$, invariant by $F_{0}$.

Next, consider a perturbed twist map $F_{\varepsilon}$, and let $\mathcal{L}_{\varepsilon}=\mathcal{L}_{0}+\varepsilon \mathcal{L}_{1}+\mathrm{O}\left(\varepsilon^{2}\right)$ be the twist generating function of $F_{\varepsilon}$. For $0<|\varepsilon| \ll 1$, there exists a hyperbolic fixed point $z_{\varepsilon}^{\infty}$ of $F_{\varepsilon}$, close to $z_{0}^{\infty}$, and it is not restrictive to normalize the twist generating function by imposing $\mathcal{L}_{\varepsilon}\left(x_{\varepsilon}^{\infty}, x_{\varepsilon}^{\infty}\right)=0$, where $x_{\varepsilon}^{\infty}=\pi\left(z_{\varepsilon}^{\infty}\right)$. In particular, $\mathcal{L}_{1}\left(x_{0}^{\infty}, x_{0}^{\infty}\right)=0$, where $x_{0}^{\infty}=\pi\left(z_{0}^{\infty}\right)$. We now define the Melnikov potential in the same way as for planar twist maps [5]:

$$
L: \Lambda \longrightarrow \mathbb{R}, \quad L(z)=\sum_{k \in \mathbb{Z}} \mathcal{L}_{1}\left(x_{k}, x_{k+1}\right), \quad x_{k}=\pi\left(z_{k}\right), z_{k}=F_{0}^{k}(z), z \in \Lambda .
$$

The Melnikov theory is based on the following properties of the Melnikov potential [4]:

$-L: \Lambda \rightarrow \mathbb{R}$ is well-defined, smooth and invariant under the action of the unperturbed map: $L \circ F_{0}=L$. Consequently, $L$ can be defined on the reduced separatrix $\Lambda^{*}=\Lambda / F_{0}$.

- The differential of the Melnikov potential $M=\mathrm{d} L$ (called the Melnikov function), measures, in first order in $\varepsilon$, the distance between the perturbed invariant manifolds, and is also defined on the reduced separatrix $\Lambda^{*}$.

- If $L \not \equiv$ constant, then the perturbed invariant manifolds $\mathcal{W}_{\varepsilon}^{\mathrm{u}, \mathrm{s}}$ split for $0<|\varepsilon| \ll 1$, i.e., they do not coincide.

- If $L$ has a critical point at $z=z_{0}$ then, for $0<|\varepsilon| \ll 1, \mathcal{W}_{\varepsilon}^{\mathrm{u}, \mathrm{s}}$ intersect transversally on a homoclinic point near $z_{0}$.

- If the unperturbed invariant manifolds are completely doubled (i.e., $\Sigma=\left\{z_{0}^{\infty}\right\}$ ), the reduced separatrix is a compact $n$-dimensional manifold without boundary. Actually, if $\sigma$ denotes the sign of the product of the eigenvalues with modulus greater that one of $D F_{0}\left(z_{0}^{\infty}\right)$, and $\mathbb{S}^{n-1}$ stands for the unit sphere of $\mathbb{R}^{n}$, then $\Lambda^{*}$ is homeomorphic to 
$\mathbb{S} \times \mathbb{S}^{n-1}$ for $\sigma=+$, and it is somewhat more complicated for $\sigma=-$ (for more details, see [4]).

In this situation, Morse theory applied to the Melnikov potential, thought as a function over $\Lambda^{*}$, gives the minimal number of transverse homoclinic orbits, under conditions of generic position.

- There exists a variational principle, in an analogous way to the one of the planar case $[9,6]$, which establishes that the homoclinic orbits of a twist map with twist generating function $\mathcal{L}$ are the extremals of the homoclinic action

$$
W[\mathcal{O}]:=\sum_{k \in \mathbb{Z}} \mathcal{L}\left(x_{k}, x_{k+1}\right), \quad \mathcal{O}=\left(x_{k}\right)_{k \in \mathbb{Z}},
$$

and a homoclinic area can be defined for every pair of homoclinic orbits $\mathcal{O}=\left(x_{k}\right)_{k \in \mathbb{Z}}$, $\mathcal{O}^{\prime}=\left(x_{k}^{\prime}\right)_{k \in \mathbb{Z}}$, and is given by the difference of homoclinic actions $\Delta W\left[\mathcal{O}, \mathcal{O}^{\prime}\right]=$ $W\left[\mathcal{O}^{\prime}\right]-W[\mathcal{O}]$. In terms of the Melnikov potential, there is also a nice expression for the homoclinic area:

$$
\Delta W\left[\mathcal{O}, \mathcal{O}^{\prime}\right]=\varepsilon\left(L\left(z_{0}^{\prime}\right)-L\left(z_{0}\right)\right)+\mathrm{O}\left(\varepsilon^{2}\right) .
$$

We finish this survey of results with two remarks about different, but related, settings.

1. Regarding Hamiltonian flows, let $H_{\varepsilon}: T^{*} \mathcal{M} \times \mathbb{R} \rightarrow \mathbb{R}$ be a time-periodic Hamiltonian of period $T$, and $F_{\varepsilon}=\Psi_{\varepsilon}^{T}$ under the conditions of this section, where $\Psi_{\varepsilon}^{t}(z)$ is the solution of the associated Hamiltonian equations with initial condition $z$ at $t=0$. If $H=H_{0}+\varepsilon H_{1}+\mathrm{O}\left(\varepsilon^{2}\right)$, one can see [4] that the Melnikov potential takes the form (already known to Poincaré)

$$
L(z)=-\int_{\mathbb{R}} H_{1}\left(\Psi_{0}^{t}(z), t\right) \mathrm{d} t,
$$

where $H_{1}$ is determined by imposing $H_{1}\left(\Psi_{0}^{t}\left(z_{0}^{\infty}\right), t\right) \equiv 0$, or simply $H_{1}\left(z_{0}^{\infty}, t\right) \equiv 0$, if $H_{0}$ is autonomous.

2. In relation with a non-symplectic setting, let us assume now that $\mathcal{M}=\mathbb{R}^{n}$, that is, $T^{*} \mathcal{M}=\mathbb{R}^{2 n}$. In that case, using a different point of view $[13,2]$, one can assume that the unperturbed map $F_{0}: \mathbb{R}^{2 n} \rightarrow \mathbb{R}^{2 n}$ possesses $n$ independent first integrals $H_{1}, \ldots, H_{n}$ on the separatrix $\Lambda$ (not necessarily in involution, since this concept requires a symplectic structure), and consider a perturbation $F=F_{0}+\varepsilon F_{1}+\mathrm{O}\left(\varepsilon^{2}\right)$ (not necessarily symplectic). Then, the Melnikov vectorial function $M: \Lambda \rightarrow \mathbb{R}^{n}$ can be written as (compare with the planar case in [5]):

$$
M=\left(M_{1}, \ldots, M_{n}\right)^{\top}, \quad M_{j}(z)=\sum_{k \in \mathbb{Z}}\left\langle\nabla H_{j}\left(z_{k+1}\right), F_{1}\left(z_{k}\right)\right\rangle, \quad z_{k}=F_{0}^{k}(z), z \in \Lambda .
$$

\section{The example}

Let us consider central standard-like maps on $\mathbb{R}^{2 n}=T^{*} \mathbb{R}^{n}$, that is,

$$
F_{0}(x, y)=\left(y,-x+\nabla V_{0}(y)\right) \quad \text { or } \quad \mathcal{L}_{0}(x, X)=-\langle x, X\rangle+V_{0}(X)
$$

where $V_{0}(x)=V_{\mathrm{c}}\left(\|x\|^{2}\right)$ for some function $V_{\mathrm{c}}:[0, \infty) \rightarrow \mathbb{R}$. Then, the "angular momenta" $A_{i j}(x, y)=x_{i} y_{j}-x_{j} y_{i}$ are first integrals and the $(n+1)$-dimensional manifold in $\mathbb{R}^{2 n}$ of zero angular momenta is $\mathcal{A}_{0}^{n+1}:=\left\{(x, y): A_{i j}(x, y)=0\right\}=\left\{(q a, p a): a \in \mathbb{S}^{n-1},(q, p) \in \mathbb{R}^{2}\right\}$. 
We now introduce the reduced map in $\mathcal{A}_{0}^{n+1}$ of $F$, as the planar standard-like map $f: \mathbb{R}^{2} \rightarrow \mathbb{R}^{2}$ defined by $f(q, p)=\left(p,-q+2 V_{\mathrm{c}}^{\prime}\left(p^{2}\right) p\right)$. We note that

$$
f(q, p)=(Q, P) \Longleftrightarrow F(q a, p a)=(Q a, P a), \quad \forall(q, p) \in \mathbb{R}^{2}, a \in \mathbb{S}^{n-1},
$$

so that the non-trivial dynamics on the separatrix is induced by the reduced map.

To take advantage of the results for planar twist maps in the lecture [5], we introduce now the McLachlan map [10] as the central standard-like map with potential $V_{0}(y)=$ $\mu \ln \left(1+\|y\|^{2}\right), \mu>1$. It has the expression

$$
F_{0}(x, y)=\left(y,-x+\frac{2 \mu y}{1+\|y\|^{2}}\right), \quad \mu>1 .
$$

Its reduced map is nothing else but the McMillan map [11] whose separatrix $\Gamma=\Gamma^{+}$has the following natural parameterization [3]:

$$
\Gamma=\left\{z_{0}(t)=\left(q_{0}(t), p_{0}(t)\right)\right\}, \quad q_{0}(t)=p_{0}(t-h), \quad p_{0}(t)=\sinh h \operatorname{sech} t,
$$

where $h>0$ is determined by the equation

$$
\cosh h=\mu \text {. }
$$

Now, it is easy to check that

1. The origin is a hyperbolic fixed point of $F_{0}$, and $\operatorname{Spec}\left[D F_{0}(0)\right]=\left\{\mathrm{e}^{ \pm h}\right\}$.

2. The invariant manifolds of $F_{0}$ are completely doubled, and the separatrix is given by

$$
\Lambda=\left\{(q a, p a):(q, p) \in \Gamma, a \in \mathbb{S}^{n-1}\right\} .
$$

3. The function $z_{0}: \mathbb{R} \times \mathbb{S}^{n-1} \longrightarrow \Lambda$ given by

$$
z_{0}(t, a)=\left(p_{0}(t-h) a, p_{0}(t) a\right), \quad p_{0}(t)=\sinh h \operatorname{sech} t,
$$

is a natural parameterization of the separatrix, i.e., $z_{0}$ is a diffeomorphism that satisfies $F_{0}\left(z_{0}(t, a)\right)=z_{0}(t+h, a)$, for $t \in \mathbb{R}$ and $a \in \mathbb{S}^{n-1}$.

As expected, we now consider a general perturbation of (4) that preserves the standard character, i.e.,

$$
F_{\varepsilon}(x, y)=\left(y,-x+\frac{2 \mu y}{1+\|y\|^{2}}+\varepsilon \nabla V(y)\right), \quad \mu>1, \varepsilon \in \mathbb{R},
$$

with $V: \mathbb{R}^{n} \rightarrow \mathbb{R}$ determined by imposing $V(0)=0$. The generating function of $F_{\varepsilon}$ that vanishes at the origin is $\mathcal{L}_{\varepsilon}=\mathcal{L}_{0}+\varepsilon \mathcal{L}_{1}$, where $\mathcal{L}_{0}(x, X)=-\langle x, X\rangle+\mu \ln \left(1+\|X\|^{2}\right)$ and $\mathcal{L}_{1}(x, X)=V(X)$. The Melnikov potential is simply

$$
L: \mathbb{R} \times \mathbb{S}^{n-1} \rightarrow \mathbb{R}, \quad L(t, a)=\sum_{k \in \mathbb{Z}} V\left(p_{0}(t+h k) a\right), \quad p_{0}(t)=\frac{\sinh h}{\cosh t} .
$$

Since $L$ is $h$-periodic in $t$ (this is the invariance of the Melnikov potential under the action of the unperturbed map), we can consider $t$ defined modulo $h$, i.e., $L$ defined over the reduced separatrix $\mathbb{S}^{1} \times \mathbb{S}^{n-1}$.

Repeating the arguments for the case of the planar twist maps [5], we see that if $V$ is a non-constant real entire function, then $V\left(p_{0}(t) a\right)$ has the same isolated singularities in the complex variable $t$ as $p_{0}(t)$, and it is not difficult to check that they remain as singularities for the Melnikov potential, which must be non-constant. In this way we have established the following result. 
Theorem 1 If $V$ is a non-constant real entire function, then the perturbed invariant manifolds of the standard-like map (6) split, for $0<|\varepsilon| \ll 1$.

\section{References}

1. S.V. Bolotin. Homoclinic orbits to invariant tori of Hamiltonian systems. Preprint, 1994. To appear in Adv. Sov. Math.

2. T.C. Bountis, A. Goriely, and M. Kollmann. A Melnikov vector for $n$-dimensional mappings. Phys. Lett. A, 206:38-48, 1995.

3. A. Delshams and R. Ramírez-Ros. Poincaré-Melnikov-Arnold method for analytic planar maps. Nonlinearity, 9(1):1-26, 1996.

4. A. Delshams and R. Ramírez-Ros. Melnikov potential for exact symplectic maps. Preprint, May 1996. To appear in Comm. Math. Phys.

5. A. Delshams and R. Ramírez-Ros and T.M. Seara. Splitting of separatrices in Hamiltonian systems and symplectic maps. In Simó [12].

6. R.W. Easton. Transport through chaos. Nonlinearity, 4:583-590, 1991.

7. H.E. Lomelí. Saddle connections and heteroclinic orbits for standard maps. Nonlinearity, 9:649-668, 1996.

8. H.E. Lomelí. Applications of the Melnikov method to twist maps in higher dimensions using the variational approach. Preprint, Univ. of Minnesota, 1993. To appear in Ergodic Theory Dynamical Systems.

9. R.S. MacKay, J.D. Meiss, and I.C. Percival. Transport in Hamiltonian systems. Phys. D, 13:55-81, 1984.

10. R.I. McLachlan. Integrable four-dimensional maps of standard type. Phys. Lett. A, 177:211-214, 1994.

11. E.M. McMillan. A problem in the stability of periodic systems. In E. Brittin and H. Odabasi, editors, Topics in modern physics, a tribute to E.V. Condon, pages 219-244. Colorado Assoc. Univ. Press, Boulder, CO, 1971.

12. C. Simó, editor. Hamiltonian Systems with Three or More Degrees of Freedom. NATO Adv. Sci. Inst. Ser. C Math. Phys. Sci. Held in S'Agaró, Spain, 19-30 June 1995. Kluwer Acad. Publ., Dordrecht, Holland, to appear in 1997.

13. J.H. Sun. Melnikov vector function for high-dimensional maps. Phys. Lett. A, 216:47-52, 1996.

14. E. Tabacman. Variational computation of homoclinic orbits for twist maps. Phys. D, 85:548-562, 1995.

15. D.V. Treschev. Hyperbolic tori and asymptotic surfaces in Hamiltonian systems. Russ. J. Math. Phys., 2(1):93-110, 1994.

Internet access:

All the authors' quoted and related preprints are available at http://www-ma1.upc.es in the Preprints pages, or at ftp://ftp-ma1.upc.es, in the pub/preprints directory. 